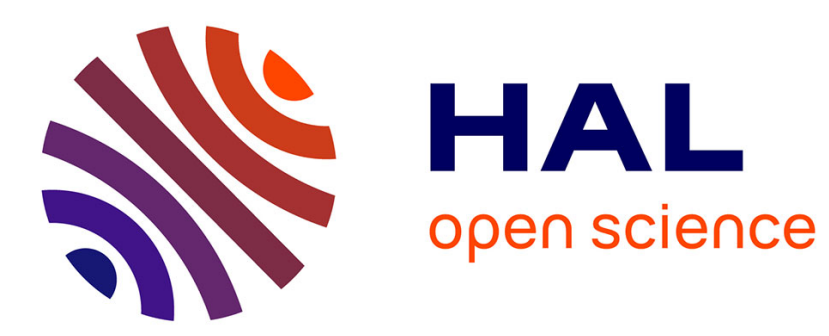

\title{
Des lieux-mouvements bien singuliers
}

Agnes Sander

\section{- To cite this version:}

Agnes Sander. Des lieux-mouvements bien singuliers. Les Annales de la Recherche Urbaine, 1996, 71, pp.44-53. hal-00520978

\section{HAL Id: hal-00520978 \\ https://hal.science/hal-00520978}

Submitted on 24 Sep 2010

HAL is a multi-disciplinary open access archive for the deposit and dissemination of scientific research documents, whether they are published or not. The documents may come from teaching and research institutions in France or abroad, or from public or private research centers.
L'archive ouverte pluridisciplinaire HAL, est destinée au dépôt et à la diffusion de documents scientifiques de niveau recherche, publiés ou non, émanant des établissements d'enseignement et de recherche français ou étrangers, des laboratoires publics ou privés. 


\section{DES LIEUX-MOUVEMENTS BIEN SINGULIERS}

Agnès Sander

Les lieux-mouvements sont situés à l'articulation entre réseaux et territoires. Leur architecture peut alors être vue comme le résultat de la combinaison des enjeux qui leur sont affectés. Ils doivent posséder un certain nombre d'attributs urbains, intégrer les contraintes de l'environnement local d'insertion, celles dues à leur nature publique et pas seulement circulatoire, et faire aussi la preuve de leur légitimité à s'implanter en un site donné.

Les aléas de la conception de ces lieux peuvent alors être décrits comme résultant d'une difficulté du gestionnaire du réseau à prendre en compte le territoire dont il ne peut totalement s'abstraire. Cette difficulté s'explique, d'abord, par la nécessité d'une similarité entre les lieuxmouvements d'un même réseau, quel que soit le site de leur implantation. Cette similarité est d'origine technique : elle permet le passage de flux, de même nature en tout point. Elle prend aussi sa source dans la volonté de rendre le service perceptible et le réseau repérable. Or, à l'opposé, le territoire n'est pas homogène : il varie en chacun de ses lieux et au cours du temps. Il s'impose avec de plus en plus de force, et porte en lui de multiples logiques. La dérive des objectifs, des intentions, des résultats, est la conséquence de cette difficulté à concilier non seulement mouvement et lieu mais aussi similarité et singularité1.

\section{La quête de cohérence du métro parisien}

Les premières stations du métro parisien ont été constituées d'une série d'espaces surdéterminés par la fonction de circulation : accès, salle des billets, couloirs et quais. Il s'agissait de canaliser les flux de piétons, afin de ne pas enrayer la bonne marche du système ${ }^{2}$. Les quais ont toujours été très valorisés ${ }^{3} ; c^{\prime}$ 'est sur eux que porte l'essentiel des rénovations. Les plus conséquentes d'entre elles sont postérieures à 1972 , lorsqu'une série de dispositifs est mise en place pour promouvoir une «politique esthétique» à la RATP. En effet, à cette date, de nombreuses stations ont déjà été transformées une ou plusieurs fois, et le résultat est un ensemble non seulement dégradé mais également hétéroclite.
Ces dispositifs aboutissent à la formulation du « parti Motte» (du nom d'un designer extérieur à la RATP), en 1974. Celui-ci réhabilite la voûte blanche, souvent dissimulée au cours des années 1960, dans les stations jugées vétustes, par de la peinture sombre. Il met en place un éclairage situé dans un bandeau linéaire bidirectionnel (qui éclaire sol et plafond), installe une banquette carrelée linéaire le long des pieds-droits des voûtes, sur

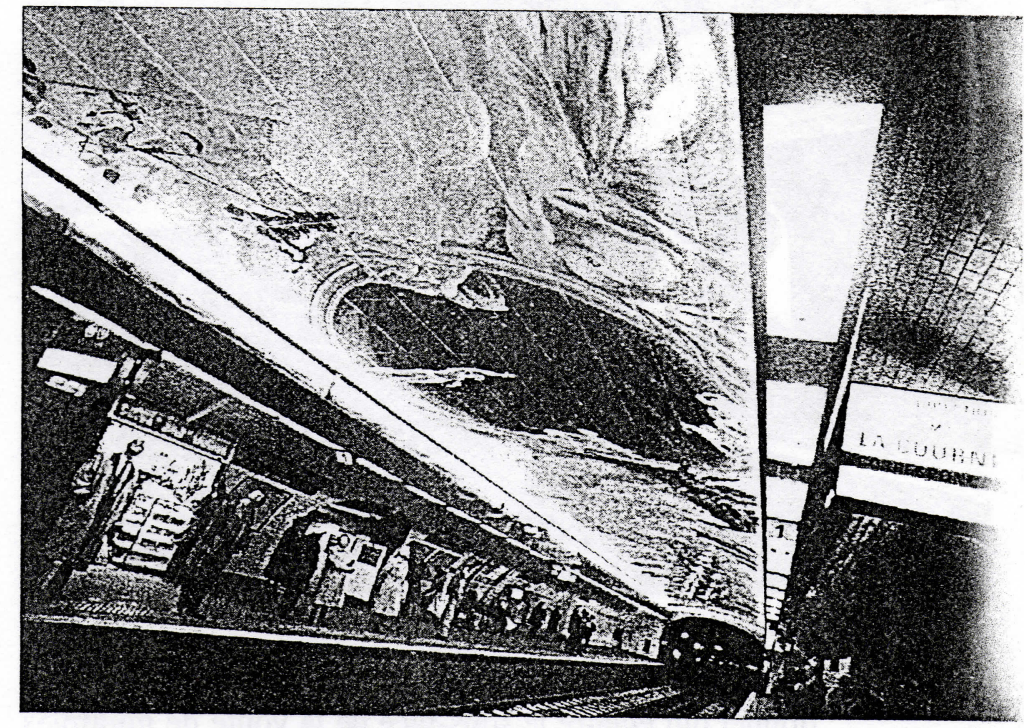

La ville est entrée dans le métro.

1. Sander Agnès, Les méthodes de conception et de production des espaces de transit : un état des lieux, GDR « Réseaux 》-CNRS, PCA, 1993, 56p. Cette étude a été suivie par Virginie Picon-Lefevre, que nous tenons à remercier ici, de même que toutes les personnes qui ont accepté de répondre à nos ques. tions, dont les noms sont cités au fil des notes.

2. Chatzis Konstantinos, Régulation des systèmes socio-techniques sur la longue durée, Thèse nouveau régime, ENPC, février 1993 ; Dekindt Jean, A propos des formes quotidiennes et actuelles de la construction du social dans le métro, RATP-Réseau 2000, 1991, 53 p. ; Pény André, "Entre ville et réseau : la station de métro", Revue d'histoire des chemins de fer, $n^{\circ} 2$, printemps 1990, p. 177-186.

3. Dupuy Gabriel, "Le réseau et la ville : le cas du métro parisien», Revue $d^{\prime}$ Histoire des Chemins de Fer, n 5-6, aut. 1992-print. 1993, p. 319-325; Lemoine Bertrand, "La voûte blanche réinventée», Architecture intérieure Créé n० 175, 1980, p. 98-102.

Les Annales de la Re't herche Urbuine $n^{\circ} 71.0180 .930-11.96 / 71 / 45 / 9$ @ ME7T 
laquelle sont disposés les sièges, armoires électriques, corbeilles... Le succès de ce parti architectural semble largement dû à la capacité du bandeau d'éclairage à concilier volonté esthétique et efficacité technique ${ }^{4}$. Il permet en effet d'obtenir un «effet d'alignement» tout en assurant la continuité du cheminement des câbles d'un tunnel à l'autre. Le rôle unificateur dévolu au bandeau d'éclairage est également rempli par la banquette : celleci permet l'intégration visuelle des multiples éléments nécessaires à la station. Le «parti Motte» constitue également une tentative d'uniformisation de l'ensemble des stations du métro par l'utilisation d'éléments similaires, déclinés dans différentes couleurs.

Les rénovations de la génération suivante sont dues au groupe «Ouï-Dire», retenu en 1988 à l'issue d'un concours. Le projet est constitué d'un bandeau bidirec-

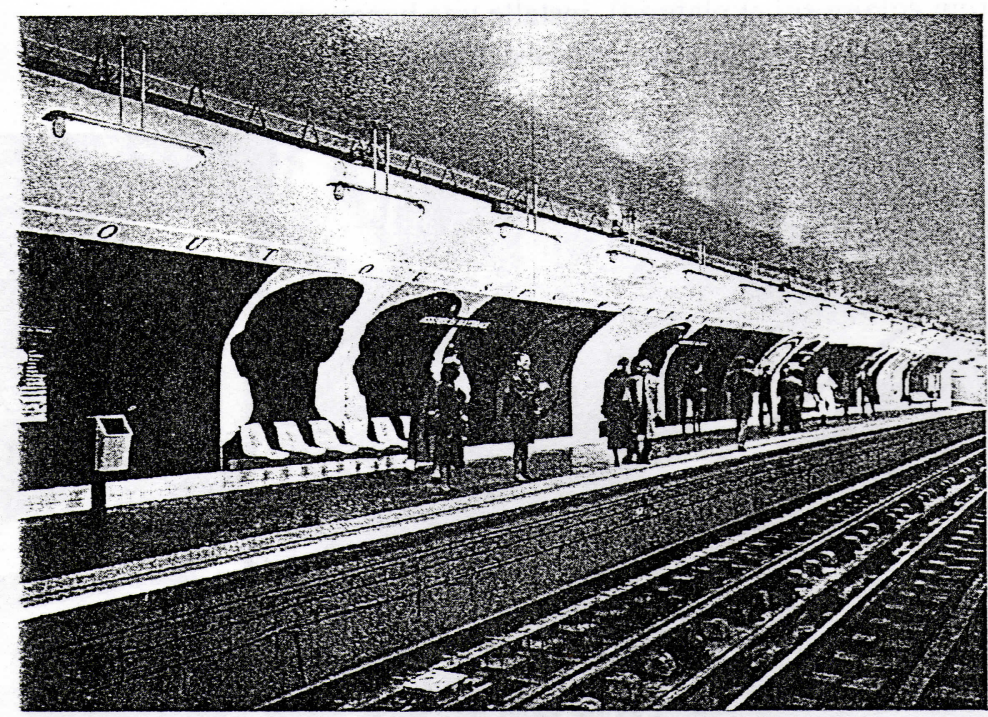

Assemblée Nationale

s

tionnel quî éclaire la sous-face de la voûte de multiples couleurs. Ce bandeau est soutenu par des éléments métalliques rassemblant les câbles. Le groupe «OuïDire» propose également des sièges bas, des appuis pour assise semi-debout, et rassemble visuellement ces éléments, accompagnés d'une corbeille à papiers, dans une composition monochrome organisée autour des affiches publicitaires.

Quelques autres expériences (Porte de Pantin et Cité) utilisent un éclairage fait d'éléments ponctuels (et non plus d'un bandeau continu), supportés par des lampadaires : l'image est celle d'un espace public extérieur ; l'aménagement de la station affirme, au moins par le signe, que «la ville est entrée dans le métro». Ces deux dernières stations font partie d'un groupe, élaboré progressivement, de stations atypiques dessinées en fonction du quartier dans lequel elles sont situées ou du nom qu'elles portent : Louvre (la première ainsi rénovée, en 1968), Assemblée Nationale, Lafayette-Chaussée

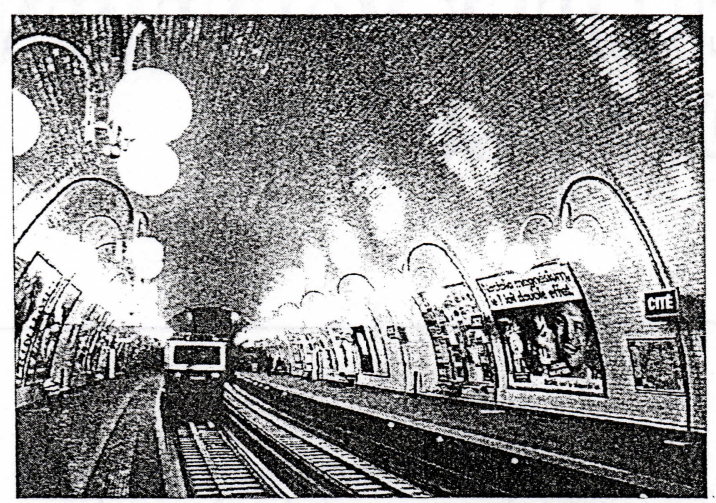

Cité

d'Antin, Arts et Métiers (la plus récente, avec un projet du dessinateur François Schuiten) $)^{5}$, etc. Les projets d'ensemble ne sont donc pas exclusifs de traitements particuliers.

Plus récemment, les couloirs, salles des billets et lieux de connexion, qui étaient jusqu'alors les grands oubliés des rénovations, ont été à leur tour réaménagés. Certaines stations (Place d'Italie, Daumesnil) expérimentent par exemple des revêtements de sol incluant un traitement particulier (couleur et forme) de la jonction entre couloirs. D'autre part, plus de trois cents stations sont actuellement «ravivées» à un rythme soutenu, ceci dans le but d'éviter que les premières ne soient déjà dégradées au moment où les dernières seront transformées.

Les choix de mise en forme des stations, à partir de 1972, peuvent être décrits en termes de «cohérence». La cohérence concerne d'abord l'intérieur de la station. Tant le «parti Motte» que celui de " Ouï-dire » cherchent à rassembler les divers objets présents sur les quais, afin de maîtriser cet espace dans sa totalité. Les aménagements visent aussi à anticiper sur de futures transformations : les éléments nouveaux devront, comme les autres, s'intégrer à l'ensemble.

Mais la cohérence est aussi recherchée entre stations. Face à un corpus hétéroclite, constitué de quais rénovés à des époques diverses, il s'agit de proposer des aménagements qui puissent s'adapter à n'importe quelle station et être implantés de manière suffisamment rapide et massive pour ne pas contribuer à la multiplication des types existants.

Enfin, l'élargissement des opérations de rénovation aux couloirs et salles des billets peut être interprété comme la recherche d'une cohérence entre les différents espaces de la station considérée comme un tout.

\footnotetext{
4. Fachard Laurent, Les lumières du métro, RATP-Réseau 2000, 1990, p. 127.

5. Ces stations sont souvent le fruit d'un partenariat entre la RATP et l'institution "de surface» concernée. "Métro : I'art underground", La vie du rail, $n^{\circ} 2290$, avril 1991, p. 11-18; "Métro, Arts et Métiers》, Architecture Intérieure Créé, $n^{\circ} 261$, oct. 1994, p. 5.
} 


\section{L'identité du VAL de Lille}

La première ligne du métro lillois a été ouverte en avril 1983. Il s'agissait d'un métro automatique : le VAL (Véhicule Automatique Léger), créé à l'origine pour desservir la ville nouvelle de l'Est lillois (d'où son sigle : «Villeneuve-d'Ascq/Lille»). La construction a été effectuée par la Communauté Urbaine de Lille (CUDL, maîtrise d'œuvre générale), puis son exploitation a été confiée au Syndicat Mixte des Transports (SMT), qui associe la CUDL et le département.

Ces remarques préliminaires ne sont pas étrangères aux choix d'architecture pour la réalisation des stations. En effet, le VAL est d'abord une affaire de prestige pour la communauté urbaine : il s'agit d'identifier les Lillois et leur métro. Il est d'autre part, pour la première fois en France, un système entièrement automatique. La nécessité de réaliser des ouvrages donnant une parfaite sensation de sécurité, en l'absence de conducteur dans les rames, se double alors de la volonté de construire des stations à l'architecture originale, toutes différentes, à l'image des quartiers de Lille et des villes de la Communauté urbaine ${ }^{6}$.

Dès l'origine, le métro de Lille a été réalisé en opposition avec le métro parisien, ce qui était possible compte tenu de son petit gabarit et du caractère nouveau de son implantation. Le métro lillois est un espace proprement réservé au transport : il se distingue fortement de l'espace public de la rue, la publicité commerciale en est

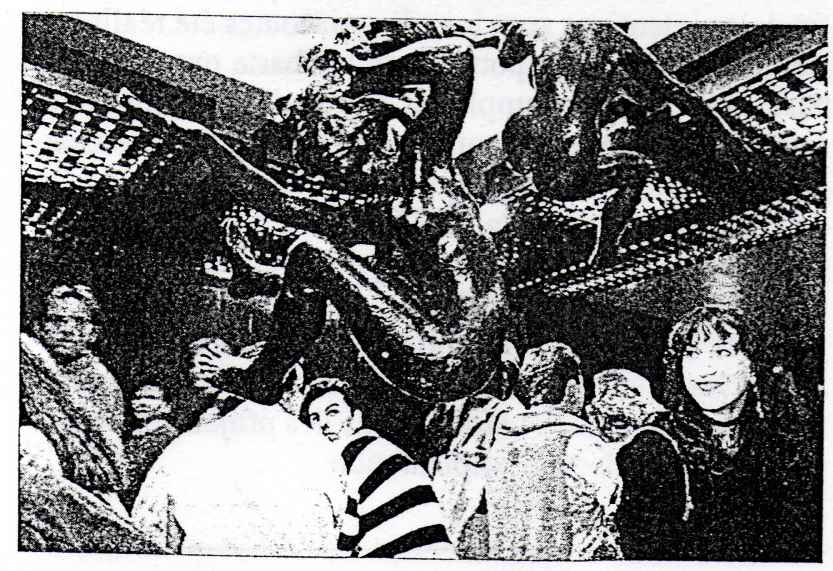

Le VAL ligne 1, station République (les Muses). Un décor intérieur évocateur du quartier traversé

exclue, il n'y a pas de commerce, les surfaces des stations sont restreintes, ce dernier point étant rendu possible par la fréquence élevée du passage des rames, autorisée par l'automatisme. Les stations ont peu de sorties (pas de «tentacules» pour traverser les carrefours en souterrain ${ }^{7}$ ), ce qui évite les longs couloirs.

Cette distinction claire entre espaces du transport et lieux publics n'empêche pas les stations de contribuer à l'aménagement de l'espace urbain : leur décor intérieur

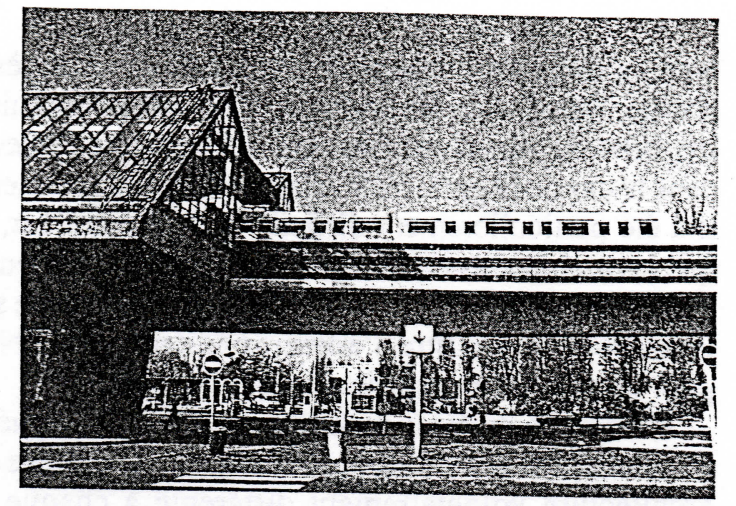

Station O. Lambert (ligne 1).

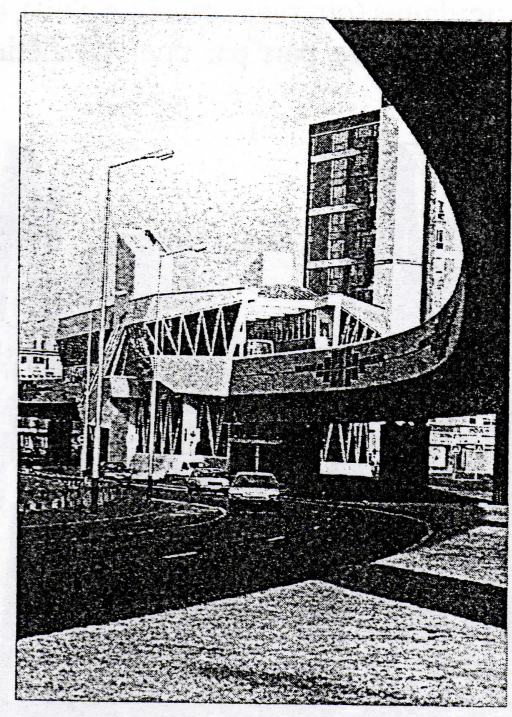

Station Porte de Valenciennes, (ligne 2).

est évocateur du quartier traversé, et certaines ont induit des aménagements de surface ${ }^{8}$. Afin d'obtenir ce résultat, chacune a été confiée à un architecte différent, chargé d'une mission restreinte d'assistance architecturale. La CUDL assure la cohérence de l'ensemble par l'intermédiaire d'un cahier des charges et grâce au suivi de chaque opération.

Compte tenu de la diversité architecturale souhaitée, l'unité est assurée par la présence, dans chaque station, d'une série d'éléments normalisés : signalétique, distri-

6. Canivet Pierre, "Le métro de la CUDL, ambiance et images", Transports $n^{\circ} 320$, déc. 1986 ; CETUR, Communauté Urbaine de Lille, 1983-1988: six ans de métro dans la communauté urbaine de Lille, 1990, p. 83.

7. Entretien avec Pierre Canivet, architecte à la CUDL en charge du VAL. Celui-ci affirme nettement son refus de suppléer en sous-sol à une urbanisation défaillante en surface : si le piéton peut traverser confortablement une rue équipée de feux de signalisation, pourquoi construire un tunnel ?

8. Knaff Bernard, "Opérations d'aménagement de surface : trois objectifs majeurs», Transports, déc. 1986, p. 641-646. Le principe de relation par la lumière entre espace souterrain et aménagement urbain extérieur est presque systématique dans le cas du métro de Toulouse, où les stations sont le plus souvent situées sous des places (et non des rues); il est également employé à Lyon. 
buteurs de billets, composteurs, rambarde d'accès à la station, signe «M» du métro... Ces éléments unitaires ont bien du mal à résister aux influences extérieures : dans le cas de la gare TGV «Lille-Europe», par exemple, il a été particulièrement difficile de les maintenir, alors que les architectes en charge de la gare souhaitaient dessiner eux-mêmes les équipements techniques et de signalétique - au même titre d'ailleurs que ceux de la SNCF9 pour les adapter à un projet jugé prestigieux.

L'exemple du VAL de Lille montre que la cohérence (formelle) entre stations peut exister dans le cadre d'une architecture volontairement différente à chaque fois ; elle est ici assurée d'une part par la présence de volumes similaires (ouvrages de génie civil), quelle que soit la station, d'autre part par une signalétique et des éléments

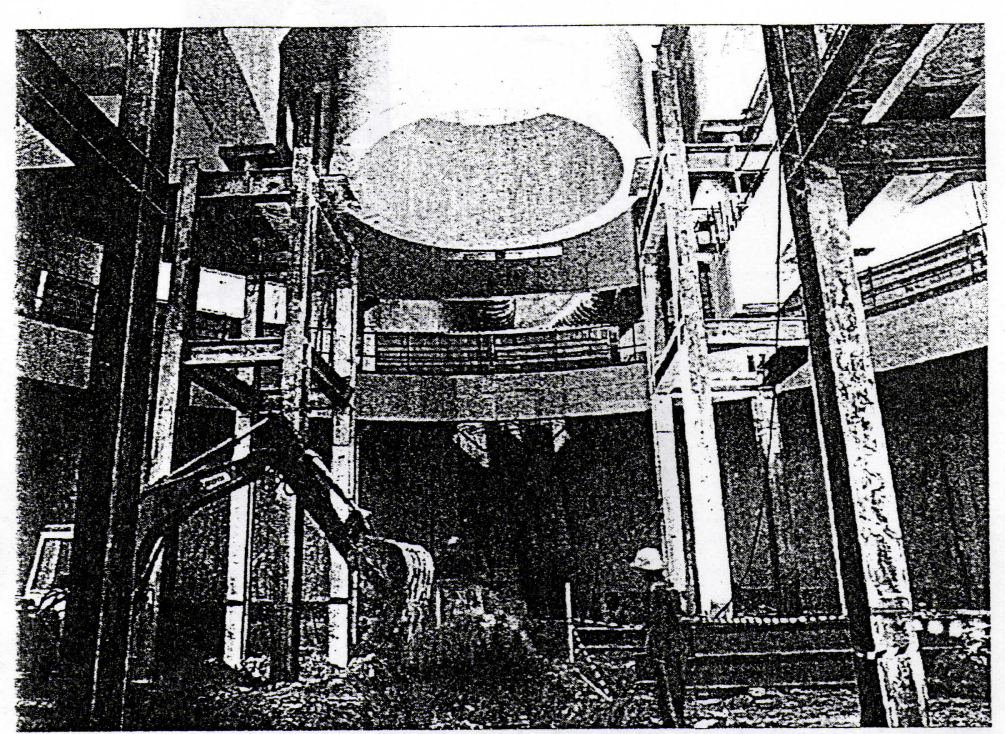

Météor. Puits de circulation verticale. Chantier station Madeleine

techniques traités partout de la même manière. Ce cas d'une infrastructure entièrement nouvelle, mais qui commence déjà à évoluer, met aussi en évidence la fragilité de la cohérence à l'épreuve du temps. Celle-ci n'est sauvegardée - pour l'instant du moins - que grâce à la forte centralisation de la gestion de l'architecture à la CUDL, qui implique un suivi très attentif des différents projets, de leur conception à leur mise en œuvre.

\section{La rigueur du projet Météor}

Le projet Météor ${ }^{10}$ (Métro Est-Ouest Rapide) de la RATP, première ligne automatique du réseau parisien, reliera en 1998 les quartiers Tolbiac, Bercy, Gare de Lyon, Châtelet et Madeleine, avant de s'étendre au-delà. Il a été saisi comme une occasion de fabriquer des stations nouvelles, pour lesquelles une intervention architecturale pouvait être envisagée dès le stade du génie civil. L'atelier d'architecture Bernard Kohn a été retenu pour l'intérêt de ses propositions d'ordre méthodologique ${ }^{11}$, visant en particulier à articuler au mieux maîtrise d'ouvrage et maîtrise d'œuvre et à repérer d'éventuelles divergences d'intentions à l'intérieur même de la maîtrise d'ouvrage.

Cette méthode de travail, qui fonctionne par allerretours successifs entre maîtrise d'œuvre et maîtrise d'ouvrage, avec apport de consultants extérieurs, a conduit à la rédaction d'une charte définissant un certain nombre de principes, parmi lesquels :

- pénétration la plus importante possible de lumière naturelle dans les espaces souterrains, (création de «puits de lumière» où sont concentrées les circulations verticales) ;

- facilitation du repérage ;

- utilisation de mezzanines pour permettre une bonne compréhension (lisibilité) des espaces ;

- diminution maximale de la longueur des couloirs ;

- création d'une «structure-intégration» (double-mur) qui accueille le plus grand nombre possible d'équipements (fluides, signalétique...).

Cette charte a été suivie de plusieurs autres documents, également soumis aux différents interlocuteurs des architectes.

Le cas de Météor illustre clairement de quelle façon un travail de conception fondé sur une méthode peut permettre de conserver une série d'intentions initiales jusqu'à la réalisation finale, en autorisant la (ré)-conciliation d'objectifs souvent contradictoires, même si, au final, les intentions sont loin d'avoir toutes été réalisées. Certains éléments importants de la charte ont ainsi disparu, comme par exemple les «puits de lumière». Ceci semble dû essentiellement à des difficultés organisationnelles et institutionnelles, qui paraissent être moins le fait de conflits internes à la RATP - ce qui témoigne d'un certain succès de la méthode - que de faiblesses dans les négociations avec les différents acteurs de l'urbanisme parisien $^{12}$. La faible légitimité du transporteur à intervenir sur les emprises extérieures à ses stations peut entraîner d'importantes transformations des projets, imposées par le contexte local d'implantation.

9. Santagio Calatrava, maître d'œuvre de l'opération, a en effet obtenu de dessiner, pour le hall de la gare, un mobilier original lui semblant plus adapté à l'ensemble de son projet que le "Kit-mobilier» de l'Atelier d'Architecture et d'Urbanisme de la SNCF. Architecture Intérieure Créé, Hors-série I, 1995, "Gares et quartiers de gares II », p. 62.

10. Cette section s'appuie essentiellement, outre des entretiens, sur : Lefèvre Frédéric, "la prise en comple des dimensions esthétiques et sensible dans les projets de la RATP», RATP-Réseau 2000, 1990, $91 \mathrm{p}$.

11. B. Kohn avait, une dizaine d'années plus tôt, fait l'effort de s'interroger sur sa pratique et de mettre en forme une méthode de "projetation" rigoureuse. Plan Construction et habitat, Processus de conception de la forme urbaine ef architecturale, réflexion sur une pratique, Bernard Kohn, architecte et urbaniste, MELT, 1986, $116 \mathrm{p}$.

12. Sur la singularité institutionnelle du cas parisien dans le domaine des transports : Lefèvre Christian, Offner Jean-Marc, Les transports urbains en question, Paris, Celse, 1990. 


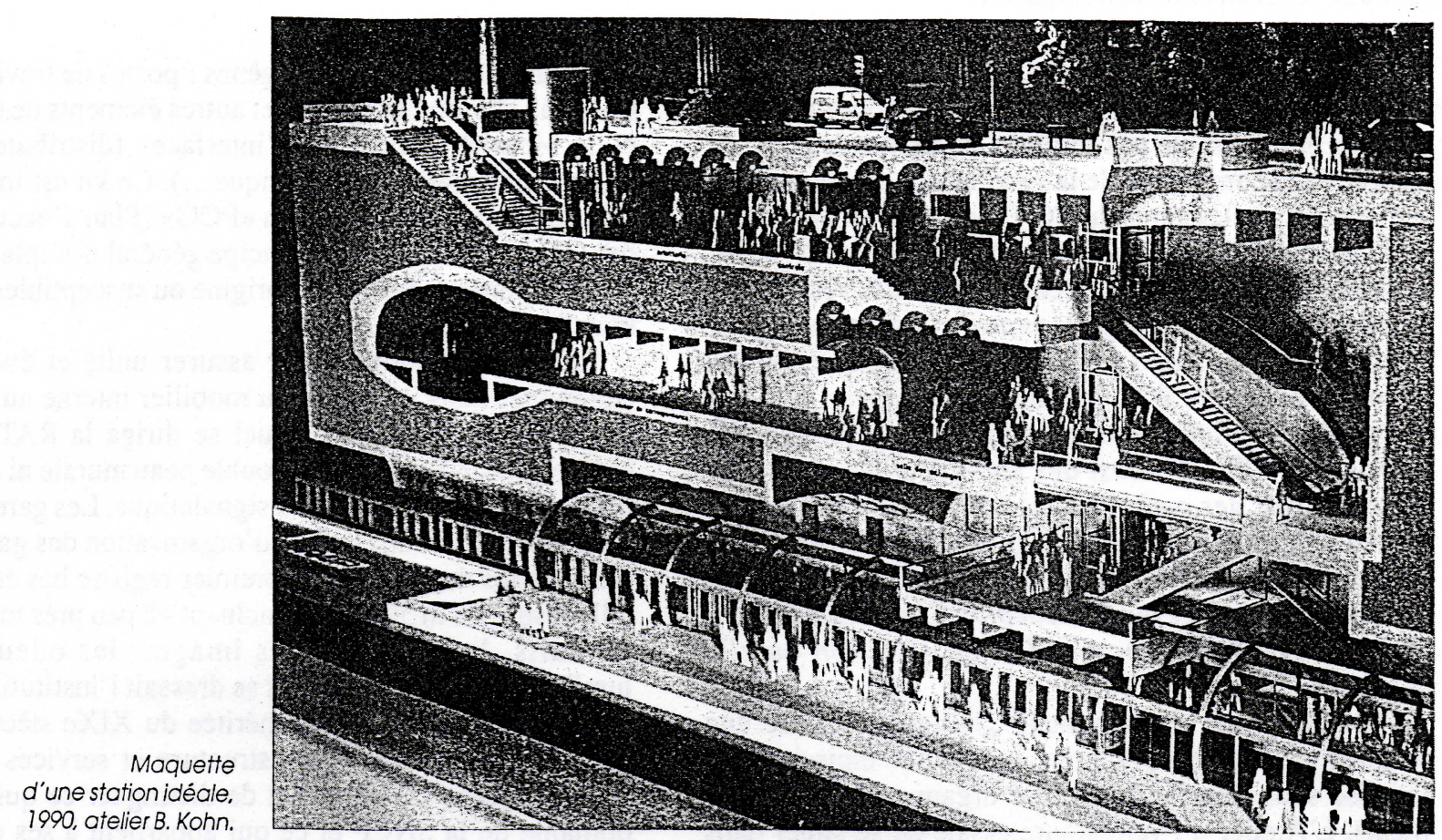

La notion de cohérence, que nous proposions d'appliquer au cas du métro parisien, semble pouvoir être utilisée - malgré ces échecs relatifs - pour qualifier certaines intentions architecturales des concepteurs des stations de Météor. La «structure-intégration » peut ainsi être assimilée aux propositions « Motte » et « Ouï-dire » de bandeaux continus. Elle a pour utilité de permettre une évolution future des équipements qui préserve homogénéité et rigueur des espaces de la station. Il s'agit donc là d'un dispositif d'intégration de la diversité et de l'hétéroclite par anticipation. Dans un contexte de restriction budgétaire, de tels éléments architecturaux pensés pour le long terme sont toutefois difficiles à imposer. Les concepteurs ignorent actuellement, par exemple, ce qui se passera s'il est un jour décidé, en contradiction avec les décisions actuelles, d'introduire des commerces dans les stations Météor, car il leur a été impossible de prévoir des installations destinées - a long terme donc - à recevoir ces éventuels commerces ${ }^{13}$. L'éventualité de l'implantation de commerces dans les lieux voués au transport n'est pourtant pas à négliger ${ }^{14}$. Par ailleurs, la «charte architecturale» vise à assurer une certaine cohérence entre les différents ouvrages réalisés (ou du moins programmés) de manière synchrone. La question de la cohérence entre les stations de Météor et celles du métro «ancien», que ce soit à l'échelle de l'ensemble du réseau ou à celle de chacun des couloirs de correspondance, subsiste néanmoins.

\section{La multimodalité des gares TGV}

Lorsqu'en 1971 est retenu le principe de la construction d'une ligne de train à grande vitesse entre Paris et Lyon, la question des gares semble devenue secondaire à la SNCF : la qualité et l'image de modernité véhiculée par le matériel roulant paraissent plus importants. Pourtant, si la construction d'une nouvelle gare à Lyon-LaPart-Dieu est décidée en 1980, c'est bien à l'occasion de l'arrivée du TGV : la SNCF avait refusé en 1967 de participer à la construction d'un tel bâtiment, souhaité par la ville depuis $1964^{15}$.

Lorsqu'est programmée dans les années 1980 la ligne du TGV Atlantique avec ses 34 gares, la SNCF a compris l'importance de l'architecture de ses bâtiments pour son image de marque. Une "charte», élaborée par l'Atelier d'Architecture de la SNCF permet alors, en conjonction avec les matériaux et couleurs utilisés (voiles tendues, bleu «atlantique», forêts de mâts...), de couvrir les zones desservies par le TGV d'édifices d'apparence similaire, facilement reconnaissables. Cette volonté d'unification visuelle du réseau n'est d'ailleurs pas limitée uniquement aux gares TGV. Le choix de concevoir les gares du futur RER parisien «Eole» (Est-Ouest Liaison Express) «en interne» rend possible une certaine identité entre ce projet et ceux des gares du TGV (les gares du réseau classique étant pour l'instant un peu oubliées...), grâce à certains choix structurels (renouer avec la tradition des grands halls de gare) et de design (mobilier, divers petits équipements...), dans le cadre d'une logique de création d'image de marque pour l'ensemble de la SNCF.

13. Entrefien avec Jean-PierreVaysse, chef de projet à l'atelier B. Kohn. 14. Stathopoulos Nikolas, Les services dans les "points-de-réseaux", RATPRéseau 2000, 1992, p. 66.

15. Société d'Equipement de la Région de Lyon, "TGV Lyon-La-Part-Dieu, pari gagné», Urbanisme, n० 198, 1983, p. 56-59. 
La voie choisie au moment de l'ouverture du TGV Atlantique reste toutefois très orientée vers des questions d'image et ne semble pas toujours permettre la prise en compte de la complexité de la gare en tant que lieu-mouvement ${ }^{16}$. Avec les gares du TGV Nord, les mots d'ordre (et la stratégie de communication) de l'Atelier d'Architecture - devenu Atelier d'Architecture et d'Urbanismede la SNCF ont changé : quand on fait une gare aujourd' hui, il ne s'agit pas de construire ce que les cheminots appellent le B.V., le bâtiment voyageur, il s'agit d'organiser l'ensemble des cheminements de la ville, des métros, des RER, des bus, des gares routières, des stations de taxis, des parkings, des hôtels avoisinants, vers les trains (...). Ce [la gare d'Austerlitz] sera l'image même d'une gare "ouverte" aux différents modes de transport, une image qui ne tient plus à sa clôture, mais à l'espace de convergence et d'interconnexion qu'elle enveloppe 17 .

Plus de façade monumentale : la gare, devenue une «entité multimodale», est constituée d'un «tapis équipé» localisé sous un grand toit, qui organise les cheminements. L'Atelier d'Architecture essaie de se situer dans une logique qui ne soit plus tout à fait celle, fonctionnaliste, de la séparation des flux : il s'agit plutôt de prévoir les déplacements possibles des voyageurs, afin de localiser aux bons emplacements les équipements appropriés.

Dans ce contexte, le design prend une importance particulière, la gare étant constituée d'éléments désolidarisés de la structure principale. Un «kit-mobilier» est élaboré par l'Atelier d'Architecture et d'Urbanisme. Il comprend des éléments capables de se combiner pour donner naissance à des séries homogènes : postes de travail destinés aux cheminots ; sièges et autres éléments de confort et de sécurité ; «mobilier d'interface» (distributeurs de billets, information, signalétique...). Ce kit est implanté sous le toit des gares selon un «POG» (Plan d'occupation des Gares) qui définit un principe général d'implantation des équipements prévus à l'origine ou susceptibles d'être installés ultérieurement.

Le principe adopté pour assurer unité et évolution cohérente (dans le temps) du mobilier interne aux gares est opposé à celui vers lequel se dirige la RATP avec Météor. A la SNCF, pas de double peau murale ni de bandeau unificateur intégrant la signalétique. Les gares TGV s'éloignent ainsi du modèle d'organisation des gares traditionnelles dans lequel un premier registre bas était installé jusqu'à $3 \mathrm{~m} 50$ du sol, incluant «à peu près tout», les produits, les services, les images, les odeurs, les lumières, alors qu'au-dessus se dressait l'institution gare et sa charpente métallique héritée du XIXe siècle ${ }^{18}$. Le parti de dissociation entre structure et services adopté dans les gares TGV permet de distinguer ce qui est du domaine de la SNCF et ce qui appartient à ses concessionnaires, autorisant ainsi un contrôle accru de ces derniers. Cette dissociation autorise également le renouvellement des éléments vieillis sans que l'ensemble ne soit touché.

Il est intéressant de souligner que des intentions très similaires (éviter la cacophonie, intégrer les éléments techniques, assurer la cohérence dans le temps) peuvent ainsi conduire à des réponses architecturales très contrastées.

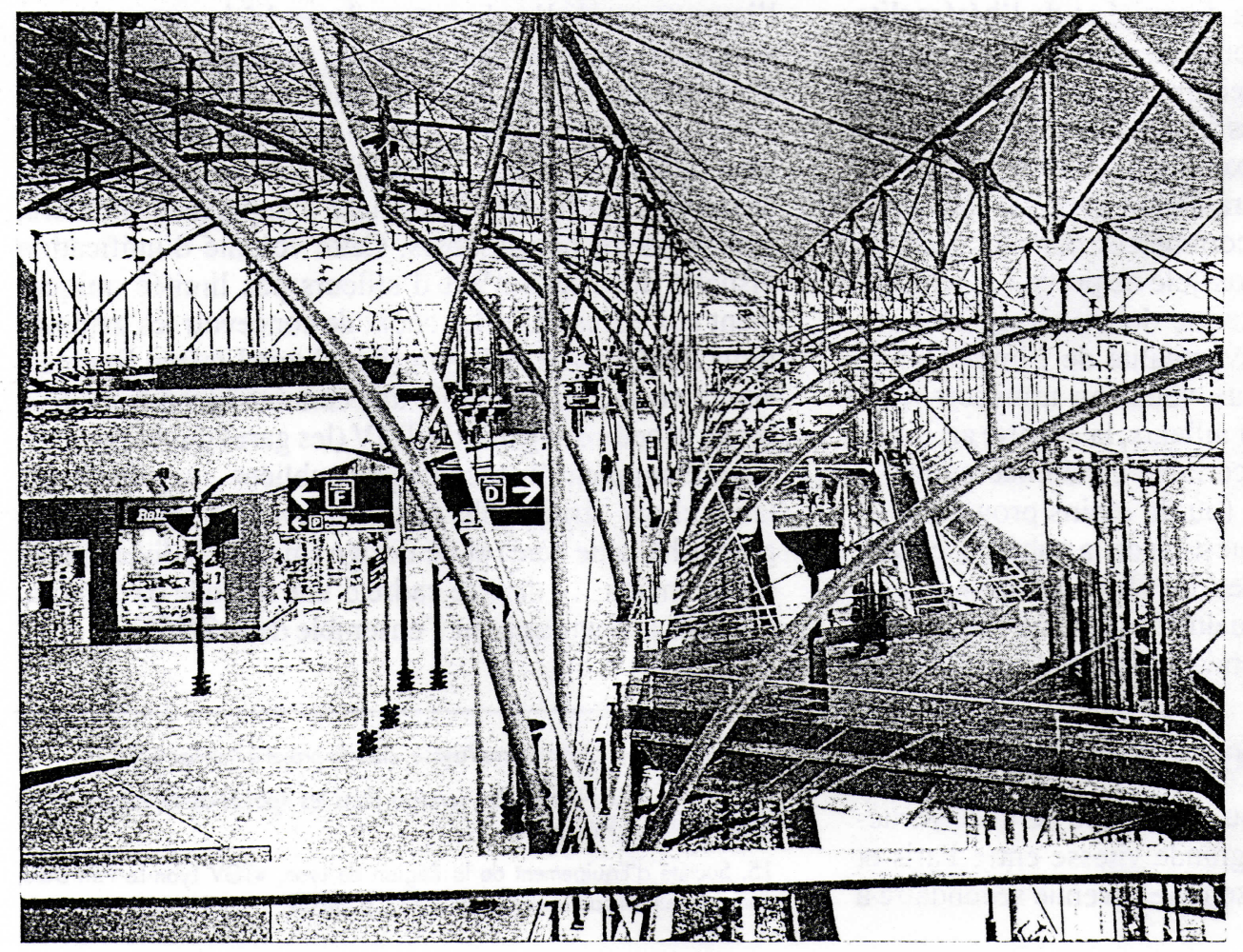

Un " tapis équipé "localisé sous un grand toit. Ici, Lille-Europe. architectes J.-M. Duthilleul, E. Tricaud, SNCF. 


\section{Les lieux-mouvements sont-ils aussi des formes urbaines?}

Si autant d'efforts sont faits pour promouvoir la cohérence de l'architecture des «lieux-mouvements», c'est que celle-ci est particulièrement difficile à obtenir (que ce soit entre les différents lieux ou à l'intérieur de chacun d'entre eux) et surtout à maintenir sur une certaine durée.

La multiplicité des acteurs en charge de l'urbanisme (distincts de ceux responsables du transport); le nombre important de ceux directement concernés par l'interconnexion des réseaux ; les doctrines architecturales et urbanistiques de séparation fonctionnelle; la prédominance du topologique sur le géométrique dans l'architecture des lieux-mouvements et, enfin, l'accumulation des strates, sont autant d'hypothèses explicatives de cette difficulté.

\section{Multiplicité des acteurs et séparation fonctionnelle}

Le cas de Météor a permis d'insister sur les difficultés de relation entre exploitants et acteurs en charge de l'urbanisme 19 . Les acteurs de l'urbanisme n'étant pas systématiquement les mêmes sur l'ensemble du territoire fonctionnel d'un réseau, les objectifs de visibilité et de lisibilité en général assignés à la conception des lieux-mouvement peuvent être contrariés par la multiplicité des sites d'implantation. La cohérence (entre points d'un même réseau) souhaitée par l'exploitant passe alors par la mise en place de dispositifs organisationnels ou institutionnels autorisant la négociation; elle passe aussi par la recherche de propositions architecturales capables de conserver leur intégrité dans n'importe quel contexte.

La séparation fonctionnelle et de gestion entre exploitants de réseaux différents et pourtant connectés en un même point est une deuxième difficulté : elle constitue une entrave majeure au bon fonctionnement des lieuxmouvements ${ }^{20}$. Elle a en particulier des conséquences d'ordre géométrique, qui peuvent entrer en conflit avec certaines pratiques : chaque élément ayant ses propres maîtres d'œuvre et d'ouvrage, des espaces interstitiels, au statut incertain, apparaissent entre les formes «dessinées» ${ }^{21}$. Ces espaces, n'appartenant à aucune institution, ne sont pas entretenus; ils représentent une entrave importante au cheminement et rendent difficile la correspondance; ils sont la trace visible dans l'espace bâti de l'absence d'une pensée globale 22 qui serait appliquée au lieu lors de sa conception.

\section{Des espaces à vocation circulatoire}

La présence de ces espaces résiduels peut également être interprétée à partir de l'hypothèse d'une suprématie, dans les lieux-mouvements, des dimensions topologique, dimensionnelle et sémiologique ainsi que du traitement de l'enveloppe (la paroi) sur la résolution géométrique. En cela, les lieux-mouvements ne sont pas des formes urbaines comme les autres.

Ils bénéficient en effet généralement d'une organisa-

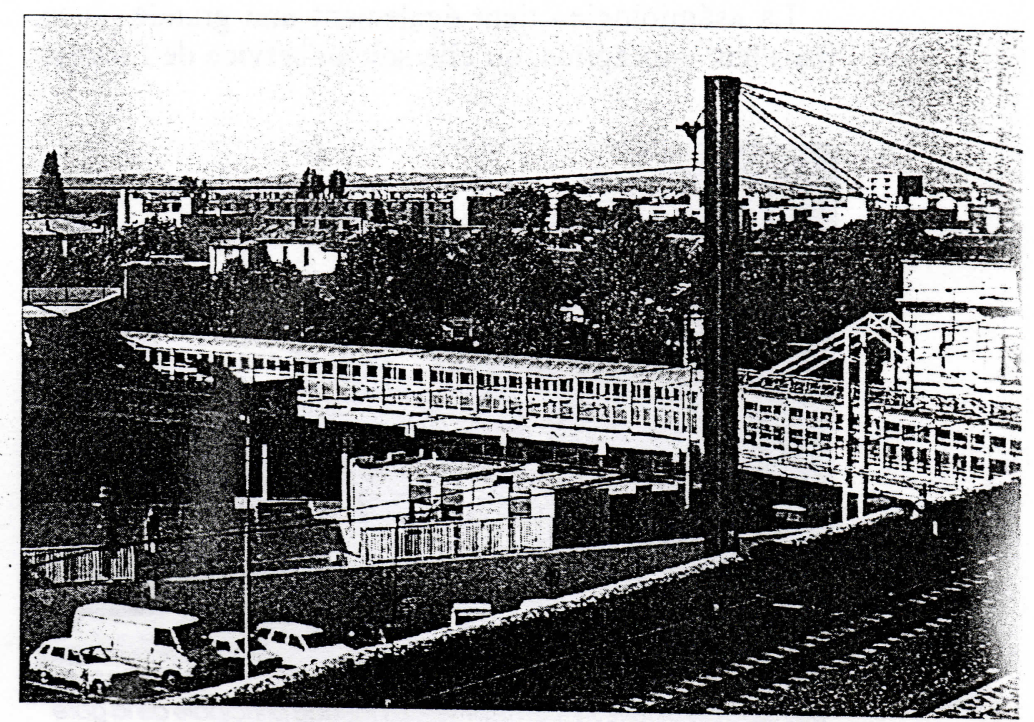

Les conséquences de la séparation fonctionnelle: entre gare ferroviaire et gare routière, un "fuyau».

tion à caractère topologique : la situation relative des différents espaces les constituant est étudiée. Ces espaces séparés fonctionnellement - sont reliés par des couloirs $^{23}$; dans le cas des infrastructures souterraines, la forme adoptée par ces «tubes» est indépendante de celle des lieux mis en relation : il n'existe aucune adaptation réciproque d'ordre géométrique. Entre les tuyaux et les volumes simples, des masses de terre s'interposent...

16. Monnier Gérard, "Architecture récente des gares : monument ou interface ?», Revue d'histoire des chemins de fer, $n^{\circ}$ 5-6, aut. 1991-print. 1992, p. $171-179$

17. Duthilleul Jean-Marie, "Connexion, lien, ouverture avec la ville, des enjeux considérables", Technopolis international, n 13, avril 1993.

18. D'après un entretien avec Christian Descamps, responsable de l'équipe "design" de l'Atelier d'Architecture et d'Urbanisme de la SNCF.

19. Voir aussi : Offner Jean-Marc, "Entreprises de réseau et territoires, manager la différence», in Curien Nicolas, Economie et management des entreprises de réseau, Economica, 1992.

20. Barouch G., Chapirot C., Le Corre B., Etude Villejuif-Lovis Aragon. Un complexe d'échanges?, RATP-Réseau 2000, 1987, 91 p.

21. Sander Agnès, "Intégration de la gare dans la ville", Revue d'Histoire des Chemins de Fer, n'5-6, aut. 1991/print. 1992, p. 149-160.

22. Devillers Christian, "Le projet urbain", Les Minis-Pa, Paris, Pavillon de l'Arsenal, $n^{\circ} 2,1994$, p. 8-47.

23. Picon-Lefebvre Virginie, "L'opération Maine-Montparnasse comme pôle de développement urbain", Revue d'Histoire des Chemins de Fer, n5-6, aut.
1991/print. 1992, p. 281-290. 
Les lieux-mouvements sont, en outre, soigneusement dimensionnés, compte tenu de la nécessité d'obtenir une bonne adéquation entre flux estimés de voyageurs et taille des dispositifs de liaison. Le dimensionnement est essentiel pour assurer la fonction circulatoire de ces lieux.

La «sémiologie» tient également une grande place dans leur conception, qu'elle soit au service de l'image

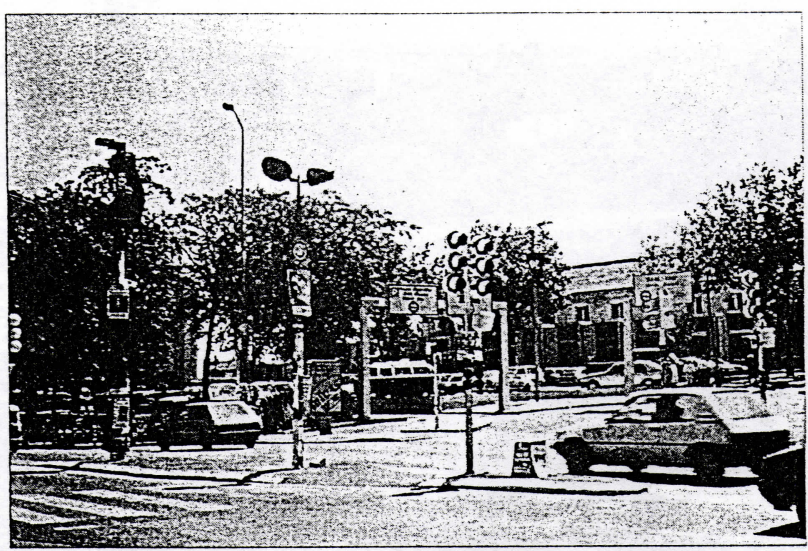

Des espaces extérieurs mal maîtrisés : La façade principale de la gare d'Avignon.

du réseau, de sa lisibilité ou des collectivités locales où l'infrastructure est implantée.

La géométrie, qui permet de qualifier les espaces grâce à un travail effectué sur leur forme même, est à l'inverse peu utilisée. Une première raison en est la valorisation du mouvement, propre aux entreprises de transport ${ }^{24}$. Cette mise en avant de la circulation peut conduire à négliger la qualité des espaces fréquentés par les usagers, s'ils sont considérés comme de simples espaces de transit. Une autre raison, qui ne concerne pas seulement les lieux-mouvements mais participe d'une évolution plus générale de l'architecture, tient à l'instrumentalisation de la notion de confort. Le confort est souvent invoqué lorsqu'il s'agit de créer des espaces chaleureux, destinés à l'attente ou à la flânerie, au sein d'un lieu-mouvement, mais son sens a évolué. Avant la Renaissance, créer un espace confortable, c'était lui donner une mise en forme agréable et unitaire ${ }^{25}$. Aujourd'hui, de plus en plus, c'est se soucier de l'enveloppe (isolation thermique, étanchéité, etc.) et du mobilier, de la qualité de l'air et de la sécurité. La géométrie n'est plus considérée comme susceptible d'apporter du confort sauf de manière très marginale.

Ceci conduit à rendre difficile la recherche de cohérence entre les différents espaces d'un même lieu-mouvement : celle-ci ne peut passer par la seule connexion topologique. Elle nécessite sans doute un arrangement géométrique de la rencontre de ces espaces, ou peut être d'autres types encore d'organisations spatiales, qui restent à inventer...

\section{L'accumulation des strates}

Mais la cohérence doit également, pour s'imposer, résister à l'épreuve du temps. Entre les premiers lieuxmouvements construits par une entreprise et d'autres plus tardifs, les doctrines esthétiques et techniques tout comme les responsables en charge des opérations ont en général changé. Le corpus résultant, à une époque donnée, s'avère en général assez hétéroclite. Il en est de même à l'intérieur de chaque lieu, les équipements techniques et la signalétique évoluant très rapidement. Les strates successives s'accumulent alors, le remplacement systématique de l'ensemble du matériel présent en gare ou en station étant impossible à réaliser en un temps suffisamment court pour que l'apparition d'un nouvel équipement ne vienne rendre cet effort inutile. Les lieuxmouvements seraient ainsi soumis à des évolutions incluant à la fois permanence et substitution. En cela, ils pourraient être considérés comme des formes urbaines plus traditionnelles.

\section{Des dispositifs à vocation régulatoire}

Les stratégies mises en œuvre par les entreprises gestionnaires pour atteindre les différents objectifs qu'elles se sont fixés et, surtout, pour les maintenir à la fois dans le temps et dans l'espace (sur plusieurs sites) sont de diverses natures (institutionnelle, organisationnelle, etc.). Mais toutes, finalement, doivent se concrétiser par la réalisation d'une forme construite. Il peut alors être intéressant d'installer des dispositifs spatiaux capables d'agir directement sur les formes, en évitant leur dispersion. Certains prônent une mise en forme intégrant dès l'origine une certaine complexité ${ }^{26}$. D'autres proposent des dispositifs destinés à réduire le désordre en l'intégrant dans une structure unitaire (Météor) ou en acceptant des éléments d'apparence disparate, pourvu qu'ils soient disposés selon une trame fixée à l'avance (tapis équipé des gares TGV). Ces dispositifs spatiaux peuvent, bien évidemment, nécessiter eux-mêmes la mise en place de procédures institutionnelles ou organisationnelles pour leur application.

Les stratégies des entreprises gestionnaires peuvent alors être vues comme des dispositifs régulatoires, visant à préserver l'intégrité des formes nécessaires au fonctionnement de l'infrastructure et du service, mais prenant aussi en compte l'irruption du local au sein du réseau; reconnaissant l'existence de singularités spatiales ; anticipant les évolutions à venir. L'histoire de la formation

24. Amar Georges, "Pour une nouvelle conception des réseaux dans la ville», Quaderni, n6, hiver 88/89, pp. 23-34.25. D’après Giedion Siegfried, La mécanisation au pouvoir, Centre Georges Pompidou, Paris, 1980, 592 p. (lère ed. 1948).

26. Venturi Robert, De l'ambiguité en architecture, Dunod, 1981. Voir aussi : Garcia Jean-Claude, Treuttel Jean-Jacques, "Architecture de l'Excès", Monuments Historiques, $n^{\circ} 6,1978$, p. 10-13. 
des lieux-mouvements est faite de la suite incessante de ces interventions : le désordre, en effet, ne se cantonne $\operatorname{pas}^{27}$. Quels que soient les dispositifs mis en place en un temps $\mathrm{T}$, d'autres actions inlassablement répétées s'imposent, afin que les résultats acquis puissent être maintenus. Or, jusqu'à présent, de telles actions n'ont eu qu'une portée limitée : les lieux-mouvements hérités sont généralement disparates, compte tenu non seulement des contraintes locales mais aussi des politiques successives des gestionnaires de réseaux eux-mêmes. Ceci implique que des interventions d'ensemble, menées en un temps très court, aient lieu de loin en loin, afin de recentrer les dérives observées.

Ce mécanisme de régulation limitée aboutissant à des évolutions, qui, à leur tour, font l'objet de nouvelles régulations tout aussi limitées, est essentiel : il permet que l'ensemble du système puisse évoluer. Si les gares, les stations et les complexes d'échanges sont des lieux aussi importants de l'espace urbain, ce n'est pas seulement parce que les déplacements sont constitutifs de nos modes de vie ; c'est aussi parce que les exploitants-gestionnaires n'ont jamais réussi à maintenir leurs réseaux dans le monde strictement fonctionnel de la mécanique des fluides.

\section{Agnès Sander}

27. Balandier Georges, Le désordre, Paris, Fayard, 1989, p. 252.

Agnès Sander est architecte DESA. Elle est enseignante à l'Ecole Spéciale d'Architecture et travaille au Laboratoire Technniques, Territoires et Sociétés (ENPCIIUPICNRS). Les études de cas présentées dans cet article ont été menées dans le cadre de sa thèse de doctorat : Les points-de-réseaux comme formes urbaines. Morphogenèse et enjeux de conception, soutenue le 11 décembre 1995 à l'Université Paris XII-Val-de-MarnelIUP et du rapport de recherches : Les méthodes de conception et de production des espaces de transit : un état des lieux, Plan Construction et Architecture/Cité-Projet, GDR «Réseaux»/CNRS, 1993. 